\title{
Functional Analysis of Human Foamy Virus Accessory Reading Frames
}

\author{
GERALD BAUNACH, BERND MAURER, HEIDI HAHN, MANUELA KRANZ, \\ AND AXEL RETHWILM* \\ Institut für Virologie und Immunbiologie der Universität, Versbacher Strasse 7, 97078 Würzburg, Germany
}

Received 19 March 1993/Accepted 16 June 1993

Foamy viruses belong to the retroviruses which possess a complex genome structure. The human foamy virus (HFV) isolate bears three open reading frames (the so-called bel genes) in the $3^{\prime}$ region of the genome which have been reported to give rise to possibly six difierent proteins via alternative splicing $(W$. Muranyi and R. M. Flügel, J. Virol. 65:727-735, 1991). In order to analyze the requirements of these proteins for HFV replication in vitro, we constructed a set of single and combinatory bel gene mutants of an infectious molecular clone of HFV. The mutant which lacked the transacting activator, bel-1, was found to be replication incompetent. All other mutants replicated equally well and gave rise to comparable titers of infectious cell-free virus. When HFV proviruses were put under the control of a heterologous promoter (simian virus 40), none of the accessory gene products was found to be required for expression of structural (gag) proteins. There was no evidence for a posttranscriptional regulatory protein that is present in other complex retroviruses.

The foamy viruses are far less well characterized than other subgroups of retroviruses (9). The human foamy virus (HFV) isolate of Achong et al. (1) has been molecularly cloned and sequenced $(10,22,31)$. The presence of accessory reading frames in addition to gag, pol, and env (10), evidence of a complex transcription pattern (23), and the identification of a virus-encoded transactivator of long terminal repeat (LTR)-directed transcription $(16,32)$ indicate that foamy virus gene regulation is similar to that of lentiviruses and other complex regulated retroviruses (5).

The accessory reading frames of HFV are located in the $3^{\prime}$ region of the genome between the env gene and the 3' LTR and have been designated bel (Fig. 1A) (10). On the basis of polymerase chain reaction-mediated mapping of splice sites, six bel gene-derived proteins, namely bel-1, bel-2, bel-3, bes, bet, and beo, have been postulated (Fig. 1B) (23). However, definite proof for the existence of several of these proteins is lacking, and only the bel-1 protein has been studied in greater detail. The bel-1 gene has been reported to encode a 36,000-molecular-weight nuclear phosphoprotein (36K nuclear phosphoprotein) that acts as a transactivator of transcription on distinct U3 elements of the viral LTR $(8,16,20$, 32,38 ). By using a bel-2-specific antiserum, several proteins have been identified in infected cells (21). The most prominent of the bel-2 reactive proteins (bet) was also stained with bel-1 antiserum (21), consistent with RNA mapping (23) and cDNA cloning data (14) that revealed a splice from the bel-1 open reading frame (ORF) into the bel-2 ORF (Fig. 1B).

It has been demonstrated previously that the bel-1 genomic region is required for HFV replication (21). However, that study did not show the effect of single gene mutations but did show the lethal effect of a combinatory bel-1, bet, beo, and bel-3 mutant on viral replication. Since only the bel-1 protein has been assigned a function, we aimed to investigate the relevance of the accessory HFV reading frames for the viral replication cycle by analyzing single bel gene mutants of an infectious molecular HFV clone.

\footnotetext{
- Corresponding author.
}

\section{MATERIALS AND METHODS}

Plasmid constructions. All plasmid constructions were done by established recombinant DNA techniques (33). All constructs were characterized by restriction enzyme digestion and/or dideoxy sequencing (34).

HFV mutants. Mutants of the infectious molecular clone pHSRV (30) are shown in Fig. 1C. For construction of M1, an $X b a I$ linker (New England Biolabs) providing a stop codon in the bel-1 reading frame was inserted into the Stul site in the bel-1 gene of pEB3L (30). The 5' part of the genome was introduced into the modified PEB3L plasmid as a KpnI-AatII fragment derived from p5LGPE as described previously (30). M2 and $M 3$ were derived from $\mathrm{p} \Delta \mathrm{gpe}_{\mathrm{Bg}}$ and

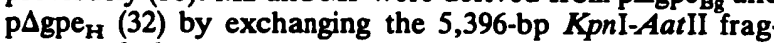
ments of these constructs with the respective 9,317-bp fragment of p5LGPE. For the mutation of overlapping reading frames in M4 to M9, BamHI-HindIII and EcoRI$X b a I$ fragments of $\mathrm{pEB} 3 \mathrm{~L}$ were introduced into the m13mp19 vector, and site-directed mutagenesis was carried out as described previously (35) by using the Amersham in vitro mutagenesis system. Mutated fragments from the $\mathrm{m} 13$ vector were then substituted for the respective fragments in pHSRV. M4 was generated by using the oligonucleotide 5'-GAGACGATCCATAGATAATGAGTC-3' (the mutated nucleotide is underlined); for construction of M5, the oligonucleotide 5'-AGGGCTACTAGAAGAGTCCAG-3' was used; for generation of M7, the oligonucleotide 5'-CATGGT TACIAAGCAGCTAT -3 ' was used; and the oligonucleotide 5'-CCTAGGATAGGAGAAGGACAT- ${ }^{\prime}$ ' was used to construct M8. M6 combines the mutations of M4 and M5, and M9 combines the mutations of M6 and M8.

HFV mutants with simian virus 40 (SV40) promoter. Starting from pSV2cat (11), the HindIII and NdeI sites were converted by linker insertion into SacI and KpnI sites, respectively. A 950-bp SacI fragment of pHSRV comprising R-U5 and some gag sequences was introduced, giving rise to pSRU5G. This plasmid was cut with NarI and AatII, and the respective 8,146-bp fragment of p5LGPE was inserted, leading to pSRU5GPE. The KpnI-AatII fragment of pSRU5GPE was then cloned into pEB3L, generating pSHFV-1; into a derivative of $\mathrm{pEB} 3 \mathrm{~L}$ lacking a 423-bp AccI-HindIII fragment 


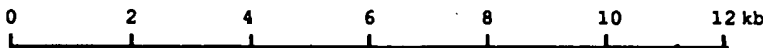

$\mathbf{A}$

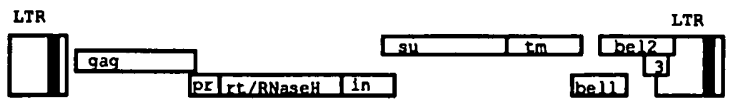

B

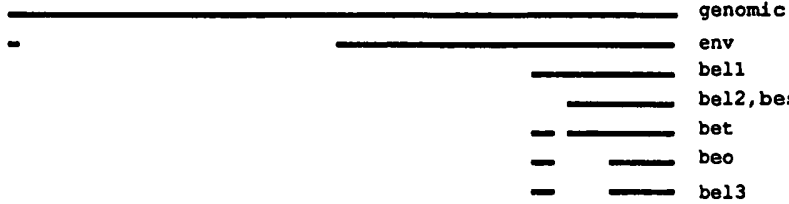

c

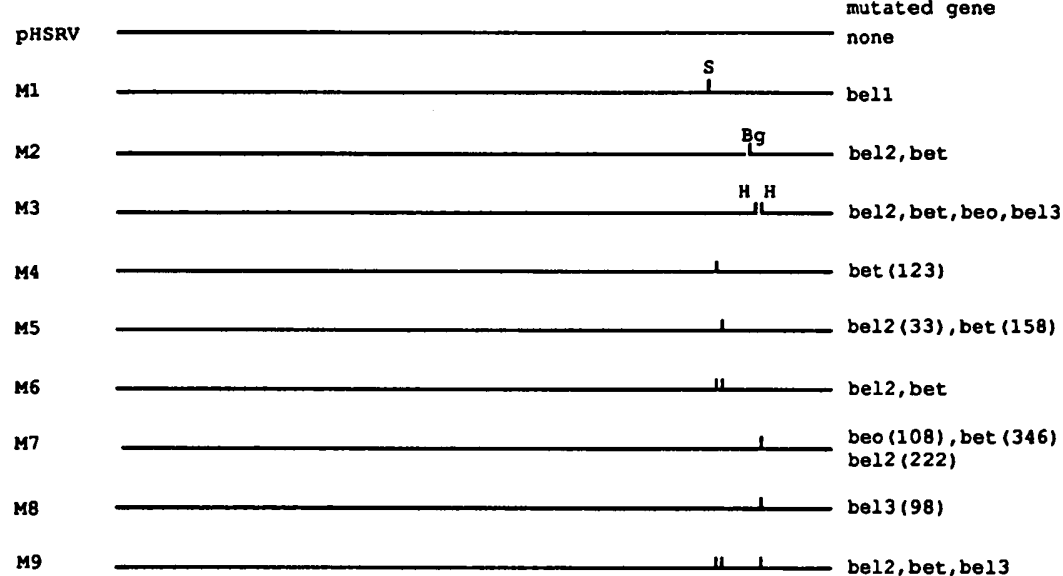

D

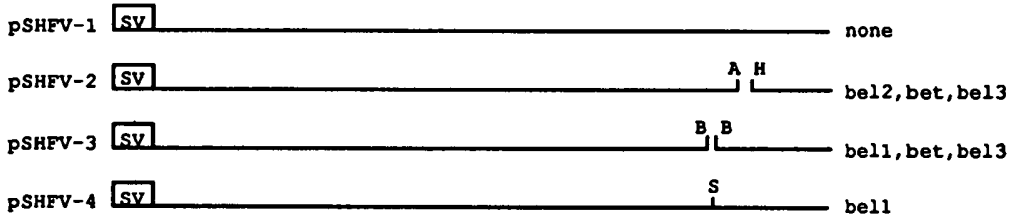

FIG. 1. Genome organization (A), transcripts (B), and virus mutants (C) of HFV and constructs with the SV40 promoter (D). For clarity, only the protein-coding exons in the bel genomic region as deduced from reference 23 are shown in panel $B$. The respective mutated open reading frames are indicated in panels $C$ and $D$. The lengths in amino acids of the bel-gene-derived proteins truncated by in vitro mutagenesis (small vertical lines) are shown in parentheses in panel C. A, AccI; B, BamHI; Bg, BgII; H, HindIII; S, StuI; SV, SV40 enhancer-promoter.

that comprises bel-2 and bel-3 sequences (to be described in detail elsewhere), resulting in pSHFV-2; and into pEB3L bearing the StuI mutation in the bel-1 gene of M1, generating pSHFV-4. pSHFV-3 was derived from pSHFV-1 by deleting a 208-bp BamHI fragment comprising bel-1 and bel-2 sequences. The pSHFV plasmids are shown in Fig. 1D.

Cells and viruses. Baby hamster kidney cells (BHK-21), human glioblastoma cells U-251 MG, and primary human embryonic lung fibroblasts (HEL) were obtained from D. Neumann-Haefelin (Freiburg, Germany), D. Bigner (Durham, North Carolina), and F. Harms (Würzburg, Germany), respectively. These cells have been shown previously to support HFV replication (30). Cells were maintained in minimal essential medium supplemented with $5 \%$ fetal calf serum, glutamine, and antibiotics. Virus derived from calcium phosphate transfection (12) of molecular clones was grown on BHK cells. Stock virus was prepared from cellfree culture supernatant (0.22- $\mu \mathrm{m}$-pore-size filtrate), aliquoted, and stored at $-70^{\circ} \mathrm{C}$ until use.

Reverse transcriptase assay. BHK cells transfected with 5 $\mu g$ of pHSRV or mutant plasmid DNA were harvested by scraping, washed with phosphate-buffered saline (PBS), resuspended in TNE $(100 \mathrm{mM}$ Tris- $\mathrm{HCl}$ [pH 7.4], $10 \mathrm{mM}$ $\mathrm{NaCl}, 1 \mathrm{mM}$ EDTA) containing $0.1 \%$ Nonidet $\mathrm{P}-40$, subjected to five cycles of freezing and thawing in dry iceethanol and $37^{\circ} \mathrm{C}$ baths, and finally centrifuged for $1 \mathrm{~min}$ at $10,000 \times g$. Protein $(25 \mu \mathrm{g})$, as determined with a commercial protein assay (Bio-Rad), was assayed in $100-\mu \mathrm{l}$ reaction mixture volumes containing $50 \mathrm{mM}$ Tris- $\mathrm{HCl}(\mathrm{pH} \mathrm{7.5),} 40$ $\mathrm{mM} \mathrm{KCl}, 1 \mathrm{mM} \mathrm{MnCl}, 0.1 \%$ Nonidet $\mathrm{P}-40,1 \mathrm{mM}$ dithiothreitol, $100 \mu \mathrm{g}$ of bovine serum albumin (BSA), $10 \mu \mathrm{M}$ dTTP, $0.1 \mathrm{mg}$ of $(\mathrm{rA})_{n}(\mathrm{dT})_{15-18}$ (Boehringer-Mannheim) per 
$\mathrm{ml}$, and $5 \mu \mathrm{Ci}$ of $\left[{ }^{3} \mathrm{H}\right] \mathrm{dTTP}$ (Amersham) for $60 \mathrm{~min}$ at $37^{\circ} \mathrm{C}$. The radioactivity of the acid-insoluble fraction was determined by scintillation spectroscopy.

Virus titration. BHK cells were seeded at a density of $2 \times$ $10^{4}$ to $3 \times 10^{4}$ cells per well into 12 -well plates. After $6 \mathrm{~h}$ for attachment of cells, $1 \mathrm{ml}$ of virus suspension was added. The virus suspension was diluted 10 -fold in culture medium from $10^{-2}$ to $10^{-7}$, and 10 wells per dilution were analyzed. The medium was changed after $16 \mathrm{~h}$, and the cells were cultivated for a further $48 \mathrm{~h}$ prior to fixing in cold methanol. For immunodetection of viral gag antigen, plates were incubated with rabbit gag2 antibody, directed against the major capsid protein p328as $(2)$ and diluted $1: 100$ in PBS containing $0.1 \%$ bovine serum albumin (BSA) for $45 \mathrm{~min}$ at $37^{\circ} \mathrm{C}$. After the plates were washed with PBS-BSA, peroxidase-coupled goat anti-rabbit antibody (Dako) was added, and the incubation was continued for $45 \mathrm{~min}$ at $37^{\circ} \mathrm{C}$. The cells were washed, and the immunostain was developed with $200 \mu \mathrm{g}$ of 3-amino9-ethylcarbazole (Sigma) per $\mathrm{ml}$ in $50 \mathrm{mM}$ sodium acetate (pH 5.0)- $0.5 \mu$ l of $\mathrm{H}_{2} \mathrm{O}_{2}$ per ml. Positive wells were counted under a light microscope. Virus titers were calculated as described previously (29).

CAT assay. The HFV effector plasmid $(10 \mu \mathrm{g})$ and $\mathrm{p} 5$ 'cat $(-777$ to +351$)$ indicator plasmid $(5 \mu \mathrm{g})(32)$ were transfected into $5 \times 10^{5}$ BHK cells as described previously (12). The DNA concentration in the transfection mix was adjusted to $40 \mu \mathrm{g} / \mathrm{ml}$ with herring sperm DNA. After $36 \mathrm{~h}$, cells were harvested, and chloramphenicol acetyltransferase (CAT) assays were performed as described previously (32) with 50 $\mu \mathrm{g}$ of protein from each transfection. Quantification of the acetylated and nonacetylated chloramphenicol on the chromatography plates was done in three independent assays with a Molecular Dynamics PhosphorImager.

Western blot and indirect immunofluorescence. The generation of the antisera directed against HFV gag and bel-1 has been described recently (2). The bel-2 antiserum was generated similarly by immunization of rabbits with a prokaryotically expressed bel-2 antigen. For antigen preparation, a carboxy-terminal 450-bp HindIII fragment of bel-2 was inserted into the bacterial overexpression vector pROS (7), and the LacZ-bel-2 fusion protein was purified as reported previously (2).

Lysates of virus-infected or transfected BHK cells were prepared after the cells were washed with PBS and resuspended in cold detergent buffer $(20 \mathrm{mM}$ Tris- $\mathrm{HCl}$ [pH 7.4], $0.3 \mathrm{M} \mathrm{NaCl}, 0.1 \%$ sodium dodecyl sulfate [SDS], $1 \%$ sodium desoxycholate, $1 \%$ Triton X-100, $1 \mathrm{mM}$ phenylmethylsulfonyl fluoride). The protein amount was measured with a commercial assay (Sigma). Equal amounts of proteins were separated by SDS- $10 \%$ polyacrylamide gel electrophoresis (SDS-PAGE) and semi-dry blotted onto nitrocellulose membrane (Schleicher \& Schuell). The membranes were blocked overnight in Tris-buffered saline ( $20 \mathrm{mM}$ Tris- $\mathrm{HCl}$ [pH 7.6], $137 \mathrm{mM} \mathrm{NaCl}$ ) containing 5\% BSA (TBS-BSA) and were washed briefty in TBS- $0.5 \%$ Tween 20 . Immunodetection was performed by incubating the membranes with rabbit antisera diluted 1:500 in TBS-BSA for 30 to $60 \mathrm{~min}$. After the membranes were washed, peroxidase-conjugated second antibody (Dako) was added at a 1:500 dilution in TBS-BSA for $60 \mathrm{~min}$. The membranes were washed, and blots were developed with the ECL chemiluminescence detection system (Amersham).

An indirect immunofluorescence assay was performed with infected HEL cells grown on coverslips and fixed in cold methanol. Slides were incubated with bel-1- or bel-2reactive or normal rabbit serum diluted 1:200 in PBS-BSA at $37^{\circ} \mathrm{C}$ for $45 \mathrm{~min}$. After the cells were washed in PBS-BSA, incubation was continued with fluorescein-conjugated second antibody (Dako). The coverslips were washed and mounted for fluorescence.

\section{RESULTS}

Design of virus mutants. The published transcript pattern of HFV suggests a variety of bel-derived proteins (23) (Fig. 1B). However, there is no information concerning the possible functions of these proteins, except for bel-1. We therefore constructed a set of mutants (Fig. 1C) of the infectious molecular clone pHSRV (32) to elucidate functional aspects of the bel proteins. M1 disrupts the bel-1 ORF by introduction of a stop codon after 106 of 300 codons without destroying any of the other reading frames. In particular, the frequently used splice donor and splice acceptor sites of the viral RNA, nucleotides 8922 and 9224 (23), respectively, remain unaffected in this construct. $M 2$ bears an 89-bp deletion upstream of a BglII site in the bel-2 ORF (32) that terminates the putative bel-2 and bet proteins after 122 and 248 codons of 356 and 482 codons, respectively. M3 harbors an in-frame 93-bp HindIII deletion of the bel-2 and bel-3 ORFs (32), which deletes codons 197 to 228 of bel-2, codons 323 to 354 of bet, and the 3' splice acceptor sites for the beo and bel-3 mRNAs, assuming that they are generated as reported previously (23) (see below). Since there is extensive overlapping of reading frames in the bel region (Fig. 1A), we introduced four mutants into the bel-2 and bel-3 ORFs by in vitro mutagenesis, thereby conserving the respective overlapping reading frame. M4 creates a stop codon in the bel-2 ORF just upstream of the first bel-2 AUG. Since the joint splice acceptor site for bel-2 and bet was reported to be located 113 nucleotides upstream of the bel-2 AUG (23), M4 terminates bet alone after 124 codons. M5 creates a stop downstream of the second bel-2 AUG, terminating bel- 2 after 33 codons and bet after 159 codons. $M 7$ is a bel-2 ORF mutant that would terminate bel-2 after 222 codons, bet after 348 codons, and beo after 109 of 242 codons. M8 is a bel-3 ORF mutant that terminates bel- 3 after 99 of 210 codons, assuming that bel-3 is expressed from a spliced mRNA that joins a bel-1 exon with the bel-3 ORF as reported previously (23). However, regarding the postulated beo and bel-3 proteins, the study of Muranyi and Flügel (23) contains an inconsistency, since they reported the translation of two proteins (beo and bel-3) having identical aminoterminal sequences but different carboxy-terminal sequences from a single mRNA (containing exon 7 and exon 10 [23]). We therefore designed M7 and M8 to cover either possibility in the generation of beo or bel-3. M6 is a double mutant combining the mutations of M4 and M5, and M9 is a triple mutant of $\mathrm{M} 6$ and $\mathrm{M} 8$.

Infectivity assay of virus mutants. The parental and mutant plasmids were transfected into BHK cells, and the cells were observed for the occurrence of typical foamy virus giant cell cytopathic effect (26) in addition to the determination of cell-associated reverse transcriptase. Cytopathic effect CPE developed in all cultures, except the M1-transfected cells. Furthermore, reverse transcriptase activity was monitored in all but the M1-transfected cultures, indicating active virus replication (Table 1). The observation of reverse transcriptase activity, in particular in cultures transfected with the mutant $M 9$, indicated that bel- 1 is the only regulatory protein required for HFV in vitro replication. When U-251 MG and HEL cells were infected with cell-free supernatants of the reverse transcriptase-positive cultures, subsequently, 
TABLE 1. Intracellular reverse transcriptase activity and titers of cell-free virus obtained with pHSRV and mutant viruses

\begin{tabular}{lcc}
\hline \multicolumn{1}{c}{$\begin{array}{c}\text { Construct } \\
\text { (ORF mutated) }\end{array}$} & $\begin{array}{c}\text { RT activity } \\
\left(10^{4} \mathrm{cpm}\right)\end{array}$ & Titer \\
\hline pHSRV & 2.13 & $4.3 \times 10^{5}$ \\
M1 (bel-1) & 0.06 & $\mathrm{ND}^{\mathrm{c}}$ \\
M2 (bel-2, bet) & 1.59 & $2.9 \times 10^{5}$ \\
M3 (bel-2, bet, bel-3, beo) & 5.33 & $1.1 \times 10^{6}$ \\
M4 (bet) & 3.72 & $2.2 \times 10^{6}$ \\
M5 (bel-2, bet) & 1.62 & $8.2 \times 10^{5}$ \\
M6 (bel-2, bet) & 5.77 & $\mathrm{ND}$ \\
M7 (bel-2, bet, beo) & 1.26 & $\mathrm{ND}$ \\
M8 (bel-3) & 1.86 & $1.6 \times 10^{6}$ \\
M9 (bel-2, bet, bel-3) & 2.74 & $\mathrm{ND}$ \\
\hline
\end{tabular}

a BHK-21 cells were transfected with $5 \mu g$ of plasmid DNA, and the reverse transcriptase (RT) activity was determined from cellular lysates on day 5 after transfection, as described in Materials and Methods.

transfection, as described in Materials and Methods. infection of 0.1 . Three days later, cell-free supernatants were harvested, and the titers were determined on BHK cells. Calculation of virus titers was done as described previously (29).

$c$ ND, not done.

typical foamy virus CPE developed in those cells (see Fig. 3 and data not shown).

To get a better insight in the replication competence of the virus mutants, we analyzed the titers of cell-free virus under defined conditions. Stock viruses were titrated, and BHK cells were infected at a multiplicity of infection of 0.1 . Three days after infection, cell-free supernatants were harvested, and titers were again determined. As shown in Table 1, the highest titer obtained was with the bet-minus mutant M4. However, the differences between titers were less than 10-fold; thus, they were within the range of biological variation.

Transactivation of the HFV LTR by virus mutants. For human immunodeficiency virus, it has been reported that the $v p r$ and nef gene products influence the gene expression of the homologous $\operatorname{LTR}(3,4,27)$. We therefore investigated whether HFV behaves similarly by analyzing the ability of the virus mutants to augment reporter gene expression directed by the complete HFV LTR. As shown in Table 2, only minor differences in transactivation of the HFV LTR were found between pHSRV and M2 to M9, with M9

TABLE 2. Augmentation of HFV LTR-directed gene expression by pHSRV and mutant viruses

\begin{tabular}{|c|c|}
\hline Construct (ORF mutated) & $\begin{array}{c}\text { Fold } \\
\text { activatione }\end{array}$ \\
\hline 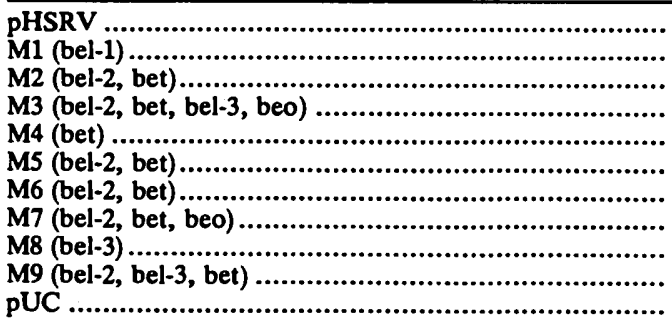 & $\begin{array}{r}36 \\
1 \\
37 \\
34 \\
51 \\
25 \\
53 \\
47 \\
36 \\
58 \\
1\end{array}$ \\
\hline
\end{tabular}

a Effector plasmids and the $\mathrm{p5}^{\prime} \mathrm{cat}(-777$ to +351$)$ indicator plasmid were cotransfected into BHK-21 cells. CAT activity in the lysates of transfected cells was determined, and the fold activation by a given effector plasmid was calculated relative to the value obtained by cotransfecting PUC DNA as a mean from three independent experiments. transactivating the HFV LTR slightly better than the parental construct or any of the other mutants. No transactivation was observed for $\mathrm{M} 1$, indicating that the mutation introduced into the bel-1 gene leads to an inactivation of the respective protein, which is consistent with results for the functional dissection of bel-1 reported recently $(14,38)$.

Stability of mutants and identification of accessory viral proteins. The aforementioned experiments did not rule out the possibility of early revertions of the point mutants. Therefore, lysates of BHK cells infected with plasmidderived stock viruses were harvested when the CPE was maximal and tested with antibodies against HFV gag, bel-1, and bel- 2 by Western blot (immunoblot).

As shown in Fig. 2A, all analyzed viruses expressed the gag precursor molecule with an apparent molecular weight of 70,000 to 74,000 and the $32 \mathrm{~K}$ major capsid protein (2). With bel-1 antiserum, two bands became visible (Fig. 2B): a band at 36,000 representing the authentic bel-1 protein $(14,21)$ and a band at 60,000 in the lanes of wild-type and pHSRV. and M8-derived viruses. Since a protein with an identical molecular weight was also stained with the bel-2 antiserum (Fig. $2 C)$, this experiment corroborates the previous finding of a bel-1/bel-2 fusion protein (bet) $(14,21,23)$. In the lanes of Fig. 2B corresponding to lysates of M2- to M5-infected cells, four novel bel-1 reactive proteins of higher electrophoretic mobility, which ideally match the truncations of the bet ORF introduced into these mutants, became visible (see above and Fig. 1C). In particular, proteins with approximate molecular weights of $14,000,18,000,28,000$, and 56,000 were observed in the lanes with lysates from M4-, M5-, M2-, and M3-infected cells, respectively. These proteins therefore represent truncated bet proteins. With the exception of the $56 \mathrm{~K}$ protein of $\mathrm{M} 3$, which has a small in-frame bet ORF deletion (see above), the other truncated proteins were not stained with our bet reactive antiserum (Fig. 2C) because the serum was generated against the carboxy-terminal bel-2 ORF.

Of particular interest were M3, M4, and M8. With mutant M3 we did not observe any difference in staining with bel-1 or bel-2 antiserum compared with wild-type- and pHSRVinfected cells, except for the truncated bet protein. Thus, we could not observe the disappearance of the postulated beo protein with a molecular weight of approximately $27,000(23)$ in M3. With mutant M4, the bel-2 antiserum did not stain the postulated $43 \mathrm{~K}$ bel- 2 protein, although this mutant leaves the bel-2 coding sequence intact and the truncated bet is processed normally. With mutant M8, again no difference appeared compared with wild-type- or pHSRV-infected cells when stained with bel-1 antiserum, as would be expected for the disappearance of the postulated bel-1/bel-3 fusion protein with a molecular weight of approximately 23,000 (23) in M8. However, there were several minor, bel-1 and bel-2 antiserum-reactive bands detectable in the lanes of those viruses which gave rise to full-length bet protein. In conclusion, the Western blot results demonstrated that the point mutations introduced into M4 and M5 were stable upon replication in vitro.

When pHSRV-infected cells were analyzed by indirect immunofluorescence assay with bel-1-reactive antiserum, we observed cells displaying nuclear fluorescence in addition to cells with predominant cytoplasmatic fluorescence as described earlier (21), while the bel-2 antiserum gave rise to only a cytoplasmic fluorescence (Fig. 3B to D). A strong nuclear fluorescence was also seen with M4-infected cells reacted with bel-1 antiserum; but no staining was observed in these cells with the bel- 2 reactive serum, again indicating 
A

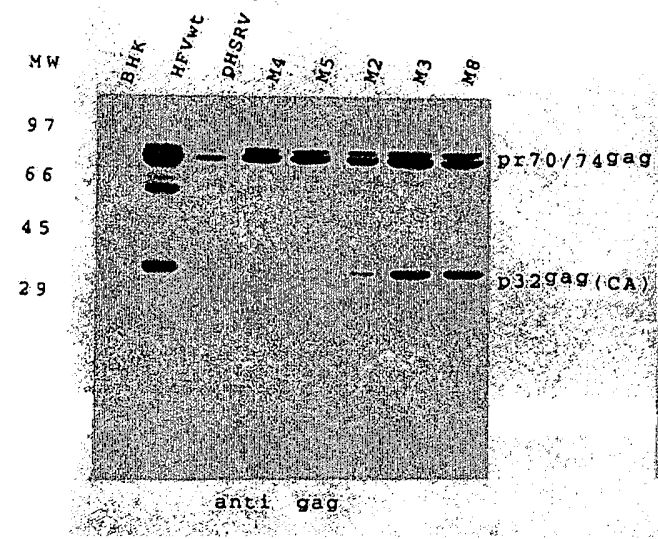

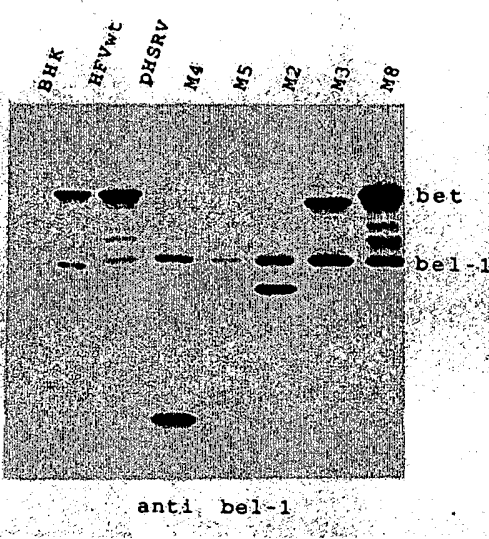

C

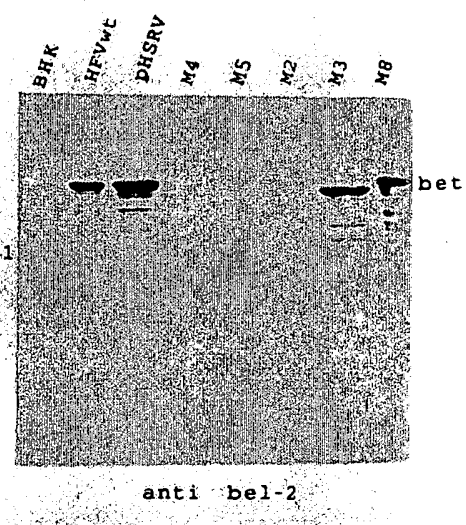

FIG. 2. Westem blot analysis with anti-gag (A)-, -bel-1 (B)-, and -bel-2 (C)-directed serum in lysates from cells infected with wild-type virus or pHSRV, M2, M3, M4, M5, or M8 transfection-derived virus. (A) The gag precursor molecules (pr70/7458) and the major capsid protein p328ag were detected in all lysates, except that from the uninfected BHK control cells. (B) bel-1 antiserum detected the 36K bel-1 protein in all lysates and the 60K bet protein in lysates from wild-type-, pHSRV-, and M8-infected cells. In M4-, M5-, M2-, and M3-infected cells, the bet proteins have reduced molecular weights of approximately 14,000,17,000,29,000, and 56,000, respectively, which are in accordance with the sizes of the mutations introduced into the bet ORF in these mutants (Fig. 1), and demonstrate the stability of the point mutants M4 and M5. (C) Lysates were reacted with antiserum directed against the carboxy-terminal bet protein. Full-length bet protein was detected in lanes with wild-type virus, pHSRV, and M8. The truncated 56K bet protein was stained in the lane of M3, which has an internal deletion, and no proteins were detected in the lanes of mutants with early truncations in the bel-2 reading frame. MW, molecular weights (in thousands), indicated on the left.

that the postulated bel-2 and beo proteins are beyond the level of detection by the immunological methods applied in this study and further demonstrating that bet was destroyed in this mutant (Fig. 3F).

No evidence for a posttranscriptional regulatory protein. Since our results indicated that the transactivator bel-1 is the only regulatory HFV protein required for replication in vitro, we investigated the possibility that bel-1 is a bifunctional protein, regulating HFV gene expression on a transcriptional and posttranscriptional level. To accomplish this, we cloned the HFV genome behind the SV40 early enhancer-promoter and introduced three mutants in the bel region, destroying either bel-1 alone or several of the postulated bel-region-derived proteins (Fig. 1D). Lysates were prepared from transfected BHK cells and probed with the gag antibody in a Western blot. The expression of gag, as well as pol and env proteins of other complex retroviruses, essentially requires the presence of cis-acting RNA elements and trans-acting regulatory proteins $(6,37)$. As can be seen from Fig. 4A, all SV40 promoter-driven constructs are able to express HFV gag. Similar results were obtained when lysates were reacted with anti-pol-directed antibodies (25) (data not shown). These results indicate that HFV does not possess a posttranscriptionally acting regulatory protein for the expression of its structural genes.

Staining of the blots with bel-1 antiserum revealed the presence of bel-1 in pHSRV, pSHFV-1, and pSHFV-2, the presence of full-length bet in pHSRV, pSHFV-1, and pSHFV-4, and the presence of a truncated bet in pSHFV-2transfected cells as expected (Fig. 4B). Reaction with bel-2 antibody revealed authentic bet protein in the lanes with lysates from pHSRV-, pSHFV-1-, and pSHFV-4-transfected cells. Consistent with the results presented above for mutant M4, no bel-2 protein was observed in PSHFV-4-transfected cells. However, there appeared several bel-1 and bel-2 reactive proteins smaller than bet in lysates of pHSRV - and
pSHFV-1-transfected cells, some of which had sizes of the calculated molecular weights of bel-2 and beo (24), but they may also represent degradation products of bet.

\section{DISCUSSION}

In this study we performed for the first time a detailed analysis of the requirement of HFV accessory reading frames for in vitro viral replication. Using point and deletion mutations in the regulatory region of an infectious molecular clone, we could show that bel-1 is the only accessory HFV protein necessary for viral replication in vitro. While bel-1 is a requisite for virus replication, our results indicate that it is only a transactivator of transcription. By assuming that no further yet-unidentified regulatory protein is expressed by HFV, our findings contrast with those for other complex retroviruses which possess two regulatory proteins, one acting on the transcriptional level and one acting on the posttranscriptional level, to express their structural genes $(5$, 6,28 ). At least in human immunodeficiency virus the necessity for the rev protein has been attributed to inhibitory sequences in the gag-pol and env mRNAs, which negatively influence the efficiency of the cytoplasmic transport and the translation of these mRNAs, unless they are complexed with rev via the rev response element $(5,6,28,36)$. The apparent lack of such a regulatory mechanism in HFV and probably in all foamy viruses may indicate that inhibitory sequences are not present in foamy virus mRNAs.

With respect to the bel-region-derived proteins (bel-1, bel-2, bel-3, bet, bes, and beo), our study shows some discrepancies from the earlier reports $(21,23)$. The postulated bel- 2 and beo proteins and the bel-1/bel-3 fusion protein could not be identified with any of our mutants. Our failure to demonstrate these proteins might be due to a lack in sensitivity of the Western blot and the immunofluorescence 

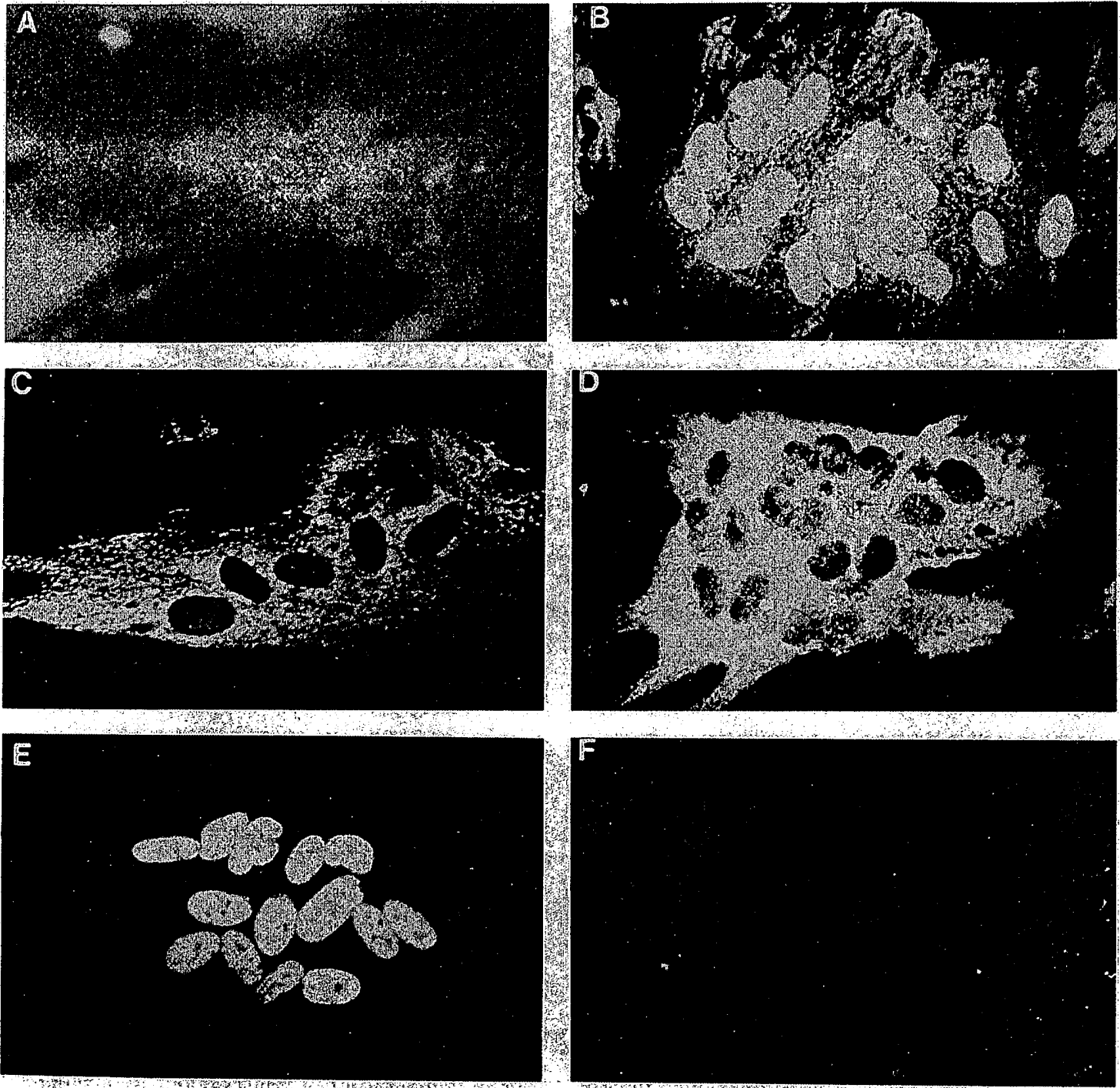

FIG. 3. Indirect immunofluorescence of primary human fibroblasts infected with pHSRV (A to D)- and M4 (E and F)-derived virus. The first antibody was from preimmune serum (A), directed against the bel-1 protein (B, C, and E) or against the bel-2 protein (D and F). Panels $A$ and $F$ have been overexposed.

compared with the polymerase chain reaction (23). Furthermore, we cannot exclude that these proteins are expressed only in certain narrow phases of the viral replication cycle, which were missed when we harvested the lysates for Western blot analysis. Further analysis, for example, kinetic studies with our mutants, may resolve this point. However, as specified above, one of the two proteins, beo or the bel-1/bel-3 fusion protein, cannot be generated as previously published

(23).

Two of the bel-region-derived proteins, bel-1 and bet (a fusion protein of bel-1 and bel-2 ORF sequences) were identified by Western blot and immunofluorescence analysis. Consistent with earlier reports, bel-1 was shown to be a 36K nuclear protein, while bet behaved like a cytoplasmic protein with an approximate molecular weight of 60,000 , slightly larger than reported previously (21). The Western blot experiments indicate that bet, although dispensable for virus replication in vitro, is an abundant viral protein. In a previous study, a $60 \mathrm{~K}$ intracellular protein was found to be among the immunodominant virus proteins when lysates of HFV-infected cells were reacted with foamy virus-positive sera of human and primate origins (24), which may indicate that bet is also expressed in the infected host. In lentiviruses some accessory virus proteins, in particular, nef, have little, if any, influence on virus replication in vitro, and their functions remain obscure $(13,18)$. However, it was shown by experiments with animals that nef as well as the vpr gene product is essential for efficient replication in vivo and induction of disease $(17,19)$. It is therefore tempting to speculate that bet promotes a similar effect, at least regard- 
A

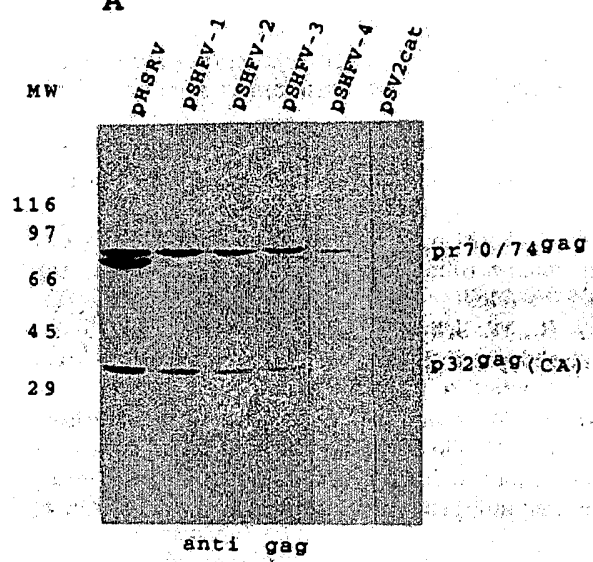

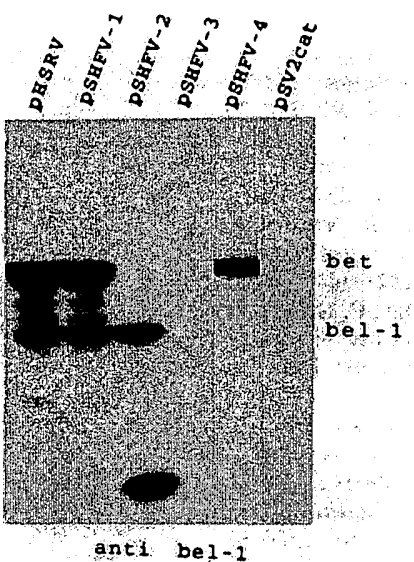

C

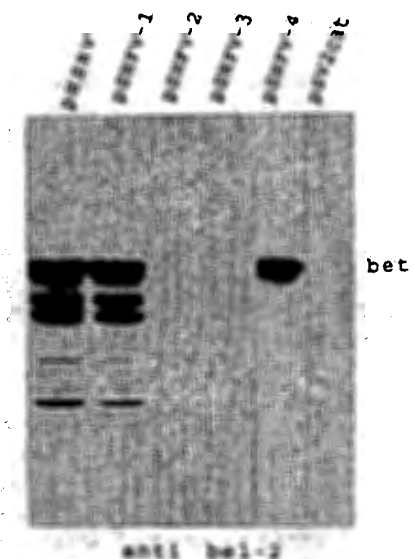

FIG. 4. Western blot analysis of lysates of BHK-21 cells transiently transfected with the infectious full-length plasmid pHSRV or with constructs in which gene expression was directed by the SV 40 promoter and which bear mutations in the bel genes (for details, see Fig. 1). Blots were developed with antisera directed against gag (A), bel-1 (B), and bel-2 (C). The detection of gag antigen in cells transfected with mutants irrespective of whether they encode any of the bel proteins is indicative of the lack of a posttranscriptionally acting regulatory protein of HFV. MW, molecular weight (in thousands), indicated on the left.

ing the virus replication characteristics, in the foamy virusinfected host.

\section{ACKNOWLEDGMENTS}

We are indebted to Lee Dunster and Sandra Bräutigam for critical review of the manuscript and Markus Czub, Adriano Aguzzi, and George Pavlakis for discussion.

This work was supported by the Deutsche Forschungsgemeinschaft (SFB 165).

\section{REFERENCES}

1. Achong, B. G., P. W. A. Mansell, M. A. Epstein, and P. Clifiord. 1971. An unusual virus in cultures from a human nasopharyngeal carcinoma. J. Natl. Cancer Inst. 46:299-307.

2. Aguzal, A., E. F. Wagner, K.-O. Netzer, K. Bothe, I. Anhauser, and A. Rethwilm. 1993. Human foamy virus proteins accumulate in neurons and induce multinucleated giant cells in the brain of transgenic mice. Am. J. Pathol. 142:1061-1072.

3. Ahmad, N., and S. Venkatesan. 1988. Nef protein of HIV-1 is a transcriptional repressor of HIV-1 LTR. Science 241:14811485.

4. Cohen, E. A., E. F. Terwilliger, Y. Jalinoos, J. Proulx, J. G. Sodrosk, and W. A. Haseltine. 1990. Identification of HIV-1 vpr product and function. J. Acquired Immune Defic. Syndr. 3:1118.

5. Cullen, B. R. 1991. Human immunodeficiency virus as a prototypic complex retrovirus. J. Virol. 65:1053-1056.

6. Cullen, B. R. 1991. Regulation of human immunodeficiency virus replication. Annu. Rev. Microbiol. 45:219-250.

7. Ellinger, S. R. Glockshuber, G. Jahn, and A. Plückthnn. 1989. Cleavage of prokaryotically expressed human immunodeficiency virus fusion proteins by factor $X_{a}$ and application in Western blot (immunoblot) assays. J. Clin. Microbiol. 27:971976.

8. Ertwein, O., and A. Rethwilm. Bel-1 transactivator responsive sequences in the long terminal repeat of human foamy virus. Virology, in press.

9. Flügel, R. M. 1991. Spumaviruses: a group of complex retroviruses. J. Acquired Immune Defic. Syndr. 4:739-750.

10. Flügel, R. M., A. Rethwilm, B. Maurer, and G. Darai. 1987. Nucleotide sequence analysis of the env gene and its flanking regions of the human spumaretrovirus reveals two novel genes. EMBO J. 6:2077-2084.
11. Gorman, C. M., L. F. Morint, and B. H. Howand. 1982. Recombinant genomes which express chloramphenicol acetyltransferase in mammalian cells. Mol. Cell. Biol. 2:1044-1051.

12. Graham, F., and A. van der Eb. 1973. A new technique for the assay of infectivity of human adenovirus 5 DNA. Virology 52:456-467.

13. Hammes, S. R., E. P. Dixon, M. H. Malim, B. R. Cullen, and W. C. Greene. 1989. Nef protein of human immunodeficiency virus type 1: evidence against its role as a transcriptional inhibitor. Proc. Natl. Acad. Sci. USA 86:9549-9553.

14. He, F., J. D. Sun, E. D. Garrett, and B. R. Cullen. 1993. Functional organization of the Bel-1 trans activator of human foamy virus. J. Virol. 67:1896-1904.

15. Hooks, J. J., and B. Detrick-Hooks. 1981. Spumavirinae: foamy virus group infections: comparative aspects and diagnosis, $p$. 599-618. In E. Kurstak and C. Kurstak (ed.), Comparative diagnosis of viral diseases, vol. 4. Academic Press, San Diego, Calif.

16. Keller, A., K. M. Partin, M. Löchelt, H. Bannert, R. M. Flügel, and B. R. Cullen. 1991. Characterization of the transcriptional trans activator of human foamy retrovirus. J. Virol. 65:2589. 2594.

17. Kestler, H. W. II, D. J. Ringler, K. Mor, D. L. Panicall, P. K. Sehgal, M. D. Daniel, and R. C. Desrosiers. 1991. Importance of the nef gene for maintenance of high virus loads and for development of AIDS. Cell 65:651-662.

18. Kim, S., K. Ikeuchi, R. Bym, J. Groopman, and D. Baltimore. 1989. Lack of a negative influence on viral growth by the nef gene of human immunodeficiency virus type 1. Proc. Natl. Acad. Sci. USA 86:9544-9548.

19. Lang, S. M., M. Weeger, C. Stahl-Henniz, C. Coulibaly, G. Hunsmann, J. Müller, H. Müller-Hermelink, D. Fuchs, H. Wachter, M. D. Daniel, R. C. Desrosiers, and B. Fleckenstein. 1993. Importance of $\mathrm{vpr}$ for infection of rhesus monkeys with simian immunodeficiency virus. J. Virol. 67:902-912.

20. Lee, K. J., A. H. Lee, and Y. C. Sung. 1993. Muitiple positive and negative cis-acting elements that mediate transactivation by bell in the long terminal repeat of human foamy virus. J. Virol. 67:2317-2326.

21. Löchelt, M., H. Zentgraf, and R. M. Flügel. 1991. Construction of an infectious DNA clone of the full-length human spumaretrovirus genome and mutagenesis of the bel-1 gene. Virology 184:43-54.

22. Maurer, B., H. Bannert, G. Darai, and R. M. Flügel. 1988. 
Analysis of the primary structure of the long terminal repeat and the gag and pol genes of the human spumaretrovirus. J. Virol. 62:1590-1597.

23. Muranyi, W., and R. M. Flügel. 1991. Analysis of splicing patterns of human spumaretrovirus by polymerase chain reaction reveals complex RNA structures. J. Virol. 65:727-735.

24. Netzer, K.-O., A. Rethwilm, B. Maurer, and V. ter Meulen. 1990. Identification of the major immunogenic structural proteins of human foamy virus. J. Gen. Virol. 71:1237-1241.

25. Netzer, K.-O., A. Schllephake, B. Maurer, R. Watanabe, A. Aguzzi, and A. Rethwilm. 1993. Identification of pol related gene products of human foamy virus. Virology 192:336-338.

26. Neumann-Haefelin, D., A. Rethwilm, G. Bauer, F. Gudat, and H. zur Hausen. 1983. Characterization of a foamy virus isolated from Cercopithecus aethiops lymphoblastoid cells. Med. Microbiol. Immunol. 172:75-86

27. Nlederman, T. M. J., B. J. Thielan, and L. Ratner. 1989. Human immunodeficiency virus type 1 negative factor is a transcriptional silencer. Proc. Natl. Acad. Sci. USA 86:1128-1132.

28. Pavlakis, G. N., and B. K. Felber. 1990. Regulation of expression of human immunodeficiency virus. New Biol. 2:20-31.

29. Reed, L. J., and H. Muench. 1938. A simple method of estimating fifty per cent endpoints. Am. J. Hyg. 27:493-497.

30. Rethwilm, A., G. Baunach, K.-O. Netzer, B. Maurer, B. Borisch, and V. ter Meulen. 1990. Infectious DNA of the human spumaretrovirus. Nucleic Acids Res. 18:733-738.

31. Rethwilm, A., G. Darai, A. Rösen, B. Maurer, and R. M. Flügel.
1987. Molecular cloning of the genome of human spumaretrovirus. Gene 59:19-28.

32. Rethwilm, A., O. Ertwein, G. Baunach, B. Maurer, and V. ter Meulen. 1991. The transcriptional transactivator of human foamy virus maps to the bel-1 genomic region. Proc. Natl. Acad. Sci. USA 88:941-945.

33. Sambrook, J., E. F. Fritsch, and T. Maniatis. 1989. Molecular cloning: a laboratory manual, 2nd ed. Cold Spring Harbor Laboratory, Cold Spring Harbor, N.Y.

34. Sanger, F., S. Nicllen, and A. R. Coulson. 1977. DNA sequencing with chain-terminating inhibitors. Proc. Natl. Acad. Sci. USA 74:5463-5467.

35. Sayers, J. R., W. Schmidt, and F. Eckstein. 1986. 5'-3' exonucleases in phosphorothioate-based oligonucleotide-directed mutagenesis. Nucleic Acids Res. 16:791-802.

36. Schwartz, S., M. Campbell, G. Nasioulas, J. Harrison, B. K Felber, and G. N. Pavlalkis. 1992. Mutational inactivation of an inhibitory sequence in human immunodeficiency virus type 1 results in rev-independent gag expression. J. Virol. 66:71767182.

37. Vaishnav, Y. N., and F. Wong-Staal. 1991. The biochemistry of AIDS. Annu. Rev. Biochem. 60:577-630.

38. Venkatesh, L. K., C. Yang, P. A. Theodorakis, and G. Chinnadural. 1993. Functional dissection of the human spumaretrovirus transactivator identifies distinct classes of dominant-negative mutants. J. Virol. 67:161-169. 